\title{
Low Temperature Effects: Surface Mount Capacitors
}

\author{
Douglas Clark \\ $8 / 17 / 92$
}

DØ Engineering Note 3740.510 -EN-335

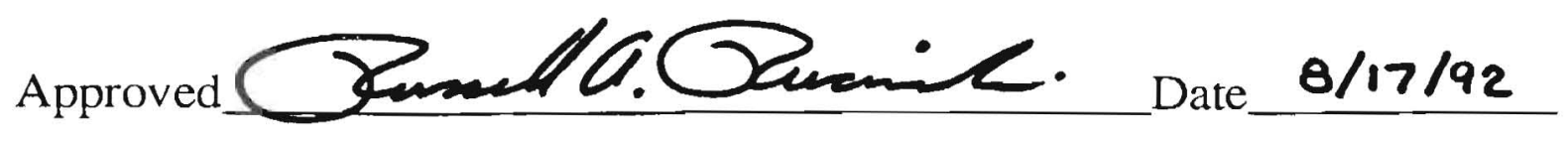




\section{Purpose and Scope}

The low signal to noise ratio produced by the VLPC (Visible Light Photon Counter) chip ${ }^{(1)}$ in the detection of a single photon necessitates the use of a filtering capacitor. Maximum performance of the filtering capabilities dictate the placement of the capacitor be as close to the VLPC chip as possible. However, the chip operates at extremely low temperatures $(7 \mathrm{~K})$. In addition, available space within the VLPC cassette is limited. Therefore it is desired to find a capacitor which provides good temperature stability at cryogenic temperatures within a minimal space (eg. surface mounted)

This engineering note presents the results from a test of the effect of low temperature on surface mounted capacitors. Preliminary testing suggested that capacitors of the tantalum type would provide the best temperature stability at cryogenic temperatures, therefore two different tantalum capacitors were tested. A ceramic capacitor was also included in the results as a comparison, even though preliminary results suggested the ceramic type would not provide sufficient temperature stability.

\section{Results and Discussion}

The three capacitors tested were:

$$
\begin{array}{ll}
\text { Tantalum \#1 } & =2.2 \mu \mathrm{F} \text { tantalum }(+/-20 \%) \\
\text { Tantalum } \# 2 & =2.2 \mu \mathrm{F} \text { tantalum }(+/-20 \%) \\
\text { Ceramic } & =1.0 \mu \mathrm{F} \text { Ceramic }(+/-20 \%)
\end{array}
$$

The capacitors were measured at three different temperatures; $4.2 \mathrm{~K}$, $77 \mathrm{~K}$, and $300 \mathrm{~K}$ achieved via liquid helium, liquid nitrogen, and ambient conditions, respectively, at atmospheric pressure. The results are plotted in figure 1. The curves represent an interpolation of the data.

In each case two identical capacitors were mounted on each card, see figure 2. The value plotted in figure 1 is thus the average of the measured value at each temperature. The maximum deviation from the average value is $0.05 \mu \mathrm{F}$.

\section{Conclusions and Recommendations}

From Figure 1 it is clear that the tantalum capacitors are quite stable 
in the temperature range from $300 \mathrm{~K}$ down to $77 \mathrm{~K}$. However, below $77 \mathrm{~K}$ the capacitance begins to diminish more rapidly. Note that the superconducting transition temperature for tantalum is $4.38 \mathrm{~K}^{(2)}$. An additional data point taken at $6 \mathrm{~K}$ (impractical during the initial test) would change the curve to reflect this transition, effectively "flattening" the curve over the temperature range $6 \mathrm{~K}$ to $300 \mathrm{~K}$. With this assumption, the tantalum type capacitors can be expected to exhibit good temperature stability at temperatures above $6 \mathrm{~K}$.

Figure 1 also shows the capacitance value of the ceramic capacitors drops of rapidly below room temperature. The curve begins to flatten below $77 \mathrm{~K}$ but the capacitance value has been reduced by two orders of magnitude from the room temperature value. The ceramic capacitors thus loses most of its ability to store well above liquid nitrogen temperatures and cannot be expected to exhibit good temperature stability at cryogenic temperatures.

\section{References}

1. Rockwell Int.

2. Scott, R. B., Cryogenic Engineering, New York: D. Van Nostrand Company, Inc., 1959. 


\section{Figure 1: Capacitance vs Temperature}

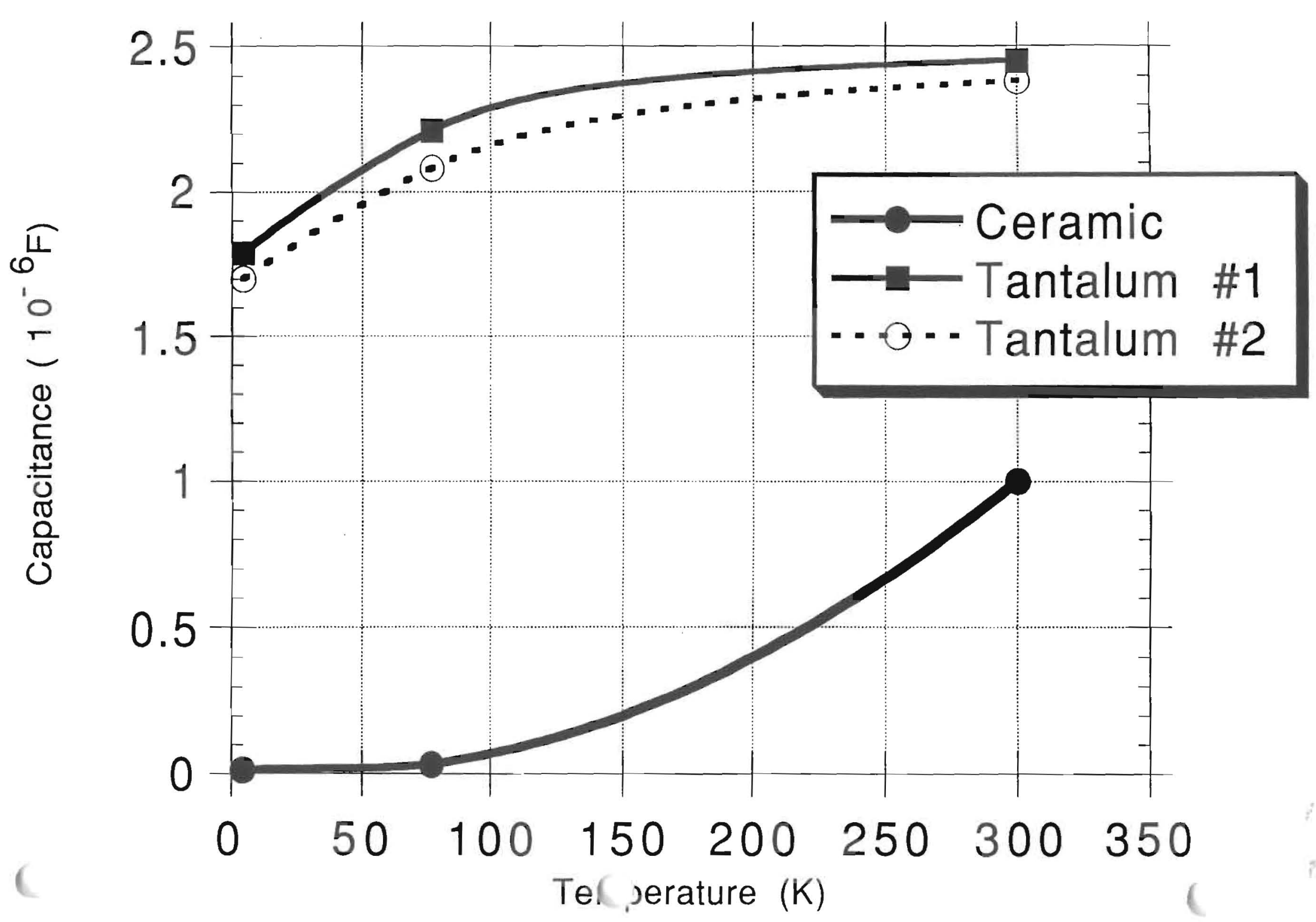






Figure 2: Low temperature Capacitance Test Samples 